OPEN ACCESS

Edited by:

Hassane Zouhal,

University of Rennes 2 - Upper

Brittany, France

Reviewed by:

Cristina P. Monteiro,

Universidade de Lisboa, Portugal Hamid Arazi

University of Guilan, Iran

${ }^{*}$ Correspondence: Hugo Corrêa

hugo.efucb@gmail.com

Specialty section:

This article was submitted to

Exercise Physiology,

a section of the journal

Frontiers in Physiology

Received: 19 October 2020 Accepted: 09 February 2021

Published: 10 March 2021

Citation:

da Silva V, Corrêa $H$, Neves $R$

Deus L, Reis A, Souza M,

dos Santos $C$, de Castro $D$,

Honorato F, Simões $H$, Moraes $M$,

Schoenfeld B, Prestes $J$ and Rosa T (2021) Impact of Low Hemoglobin on

Body Composition, Strength

and Redox Status of Older

Hemodialysis Patients Following

Resistance Training

Front. Physiol. 12:619054.

doi: $10.3389 /$ fphys.2021.619054

\section{Impact of Low Hemoglobin on Body Composition, Strength, and Redox Status of Older Hemodialysis Patients Following Resistance Training}

Victor da Silva', Hugo Corrêa ${ }^{1 *}$, Rodrigo Neves ${ }^{1}$, Lysleine Deus ${ }^{1}$, Andrea Reis', Michel Souza², Cláudio dos Santos' ${ }^{3}$, Danilo de Castro', Fernando Honorato', Herbert Simões ${ }^{1}$, Milton Moraes ${ }^{1}$, Brad Schoenfeld ${ }^{4}$, Jonato Prestes ${ }^{1}$ and Thiago Rosa ${ }^{1}$

${ }^{1}$ Graduate Program of Physical Education, Catholic University of Brasilia, Brasilia, Brazil, ${ }^{2}$ Department of Nephrology, Federal University of São Paulo, São Paulo, Brazil, ${ }^{3}$ Federal University of Tocantins, Tocantins, Brazil, ${ }^{4}$ Department of Health Sciences, University of New York City, New York City, NY, United States

Introduction: The purpose of this study was to: (i) investigate the effect of six months of resistance training (RT) on body composition, muscle strength, hematological patterns, and redox profile in maintenance hemodialysis (HD) patients, and; (ii) evaluate the effects of baseline concentrations of hemoglobin on the RT response.

Methods: One hundred fifty-seven subjects with chronic kidney disease (CKD) were randomly allocated into two groups: Control [CTL, $(n=76)]$ and RT $(n=81)$. A first visit was required for anamnesis and anthropometric measurements. Venous blood samples were collected at baseline and after twenty-four weeks of training in all patients for the analysis of clinical and redox balance markers. The RT program spanned six months and consisted of three sets of 8-12 repetitions with a rating of perceived exertion between 5 and 8 for three weekly sessions. Each exercise session was performed in twelve resistance exercises and it least for approximately $40 \mathrm{~min}$.

Results: The main results demonstrated that RT decreased waist circumference by 3\%, and decreased thiobarbituric reactive species (TBARS) by 28\%. Moreover, RT increased handgrip strength by $28.4 \%$, fat-free mass by $4.1 \%$, hemoglobin by $5 \%$, iron by $33.4 \%$, glutathione by $121 \%$, and Trolox equivalent antioxidant capacity by $14.2 \%$ ( $p<0.05$ ). Low hemoglobin concentrations impaired the effect of RT on fat-free mass gain.

Conclusion: Six months of RT in HD patients improved clinical parameters, such as hemoglobin, iron, body composition, and redox balance, while low hemoglobin concentration impaired exercise-benefits on fat-free mass in patients with CKD. These findings can contribute to a better clinical application of RT in the maintenance of hemodialysis patients.

Keywords: exercise-training, oxidative stress, muscle mass, anemia, end-stage renal disease 


\section{INTRODUCTION}

Maintenance hemodialysis patients usually present muscle wasting and a high percentage of body-fat (Cheung et al., 2010), which decreases physical functionality and increases mortality risk (Sietsema et al., 2004). The combination of increased body fat and muscle wasting aggravates chronic kidney disease (CKD) and negatively affects the health status of the patient (Jhee et al., 2020). Furthermore, these patients often develop anemia due to the lower production of erythropoietin to stabilize the production of hemoglobin, and maintenance of iron homeostasis (Astor et al., 2002; Collister et al., 2017; Gafter-Gvili et al., 2019; Wong et al., 2019). The lack of treatment for these conditions increases oxidative stress, worsening the cardio-renal axis (Kopple et al., 2001; Mcclellan et al., 2004).

Furthermore, the oxidative damage strongly stimulates eryptosis which, in turn, increases the risk to the development of anemia (Bissinger et al., 2019). Taken together, this condition might negatively affect the microcirculation leading to undesirable comorbidities as cardiovascular diseases. In this regard, treatments that improve the redox status, strength and body composition may be an important tool in the prevention and rehabilitation of anemia in CKD patients.

Thus, the development of alternative therapies to improve body composition and oxidative stress may be an important intervention with high clinical utility for CKD patients. In this context, resistance training (RT) is an exercise modality that improves fat-free mass, strength, and physical functioning in frail, older individuals, as well as those with chronic diseases (Rosa et al., 2018). However, there is a divergence in the literature concerning the effect of RT on body composition and strength improvement in this population (Rosa et al., 2018), as some studies report an increase of skeletal muscle mass (Chen et al., 2010; Song and Sohng, 2012), while others do not (Cheema et al., 2007; Kopple et al., 2007; Marinho et al., 2016).

Rosa et al. (2018) speculated that these conflicting findings may be due to the lack of progressive overload during training, and thus sought to investigate whether progressive intradialytic RT could improve body composition, physical function, and quality of life in patients undergoing regular hemodialysis. Although the study demonstrated an improvement in muscle mass, strength, and bone mineral content, there was no effect on functional capacity, handgrip strength, or self-reported quality of life in these patients after the twelveweek intervention. Nevertheless, when observing the hemoglobin levels of participants in the control and intervention groups $(11.50 \pm 1.32 \mathrm{~g} / \mathrm{dL}$ and $11.38 \pm 1.17 \mathrm{~g} / \mathrm{dL}$, respectively), some patients displayed levels $<11 \mathrm{~g} / \mathrm{dL}$, which is not recommended for this population. Stray-Gundersen et al. (2016) demonstrated that the treatment for anemia and intradialytic exercise-training produced significant improvements in peak oxygen uptake and power in CKD patients. Therefore, a possible alternative explanation for the divergent responses to RT found previously in those with $\mathrm{CKD}$ is the confounding issue of low hemoglobin concentration. Therefore, it is already known that RT might act as an alternative therapy counteracting the CKDrelated comorbidities (Corrêa et al., 2020; Moura et al., 2020;
Neves et al., 2020). Nonetheless, it remains unknown if anemia or low hemoglobin impairs the aforementioned benefits on strength, body composition, and redox balance in hemodialysis patients.

To provide clarity on the topic, we aimed to: (i) investigate the effect of RT on body composition, muscle strength, hematological patterns, and redox profile in maintenance hemodialysis patients, and; (ii) evaluate the impact of baseline concentrations of hemoglobin on RT response. We hypothesized that RT would improve body composition, muscle strength, hematological patterns, and redox profile in this population. We also hypothesized that baseline concentrations of hemoglobin would at least partly explain the divergent responses to RT observed in previous research.

\section{MATERIALS AND METHODS}

\section{Subjects}

At the beginning of the study, 202 patients volunteered for this study and were randomized by simple randomization (Suresh, 2011) in control (CTL) and resistance training (RT) group (101 patients each). In RT, 20 patients were lost to follow-up due to family issues, physical complications, and withdrawal. In CTL, 25 patients were lost to follow-up due to sleep complications, family issues, and withdrawal. Therefore, 76 patients were analyzed for CTL group and 81 for RT group (total $n=157$ ). All one hundred and fifty-seven patients undergoing maintenance phase hemodialysis were analyzed for this research investigation (age: $67 \pm 4$ years; body mass: $73.4 \pm 16 \mathrm{~kg}$; body mass index: $27.05 \pm 16 \mathrm{~kg} / \mathrm{m}^{2}$ ). All experimental protocols were approved by the local University Ethic Committee, under the number: 23007319.0.0000.0029. Written informed consent was obtained from all the participants involved in the study. Inclusion criteria for participants were: (i) age $\geq 50$; (ii) hemodialysis for at least three months; (iii) dialysis at least three times per week; and (iv) no significant medical complications in the last three months, except for vascular access correction. Exclusion criteria were as follows: (i) recent acute myocardial infarction within three months or unstable angina; (ii) systemic lupus erythematosus; (iii) congenital kidney malformation or some autoimmune disease that affects the kidneys; (iv) osteoarticular complications that could compromise physical exercise; v) decompensated

TABLE 1 | Baseline characteristics.

\begin{tabular}{lccc}
\hline Variables & CTL $(\boldsymbol{n}=\mathbf{7 6})$ & RT $(\boldsymbol{n}=\mathbf{8 1})$ & $\boldsymbol{p}$-value \\
\hline Age & $66(60-72)$ & $68(60-72)$ & NS \\
Sex, men/women & $400^{\pi / 36 \%}$ & $460^{\pi / 35 \%}$ & NS \\
Time of hemodialysis (months) & $56(35-70)$ & $29.8(15.5-51.1)$ & NS \\
Hypertension $n,(\%)$ & $75(100 \%)$ & $81(100 \%)$ & NS
\end{tabular}

Data expressed as median (minimum and maximum values).

CTL, control group; RT, resistance training; BMI, body mass index; NS, nonsignificant $(P>0.05)$.

Chi-square was applied to compare the frequencies of distribution of men/women and patients with hypertension. Mann-Whitney test was applied to compare age, weight, BMI, waist circumference, fat-free mass and time of hemodialysis from baseline characteristics. 
TABLE 2 | Loads pre- and post-intervention according to baseline status of $\mathrm{Hb}$.

\begin{tabular}{|c|c|c|c|c|}
\hline \multirow[t]{2}{*}{ Exercises } & \multicolumn{2}{|c|}{$\mathrm{RT} \mathrm{Hb}<11(n=23)$} & \multicolumn{2}{|c|}{ RT Hb > $11(n=58)$} \\
\hline & Pre & Post & Pre & Post \\
\hline e-Lastic exercises (kgf) & $23.91 \pm 7.99$ & $63.74 \pm 7.98+$ & $23.63 \pm 8.52$ & $74.6 \pm 11.57 \dagger$ \\
\hline Dumbbells exercises (kg) & $16.52 \pm 6.73$ & $34.22 \pm 4.83+$ & $15.19 \pm 6.20$ & $39.29 \pm 6.19+$ \\
\hline Weighted cuffs exercises (kg) & $20.04 \pm 6.44$ & $63.35 \pm 8.3+$ & $18.4 \pm 6.27$ & $76.1 \pm 12.61 \dagger$ \\
\hline
\end{tabular}

Data expressed by mean \pm standard deviation.

$R T$, resistance training; $H b$, hemoglobin.

Kruskall-Wallis followed by Dunns post hoc tests were applied to compare groups. ${ }^{\dagger} p<0.0001$ vs pre.

heart failure that could limit participation in training; vi) severe decompensated diabetes; and vii) severe neuropathy, retinopathy, or diabetic nephropathy. Only patients who read, agreed, and signed the written informed consent participated in this study. All participants underwent nutritional counseling with a clinical nutritionist, while the CTL group did not receive any exercise intervention. All patients received the same guidelines from a multidisciplinary team: nutritionist, psychologist, social worker, nurse, pharmacist, and nephrologist.

At study's end, we stratified the sample into groups with hemoglobin below 11g/dL $(\mathrm{Hb}<11$; CTL $n=20$ and RT $=24)$ and hemoglobin above $11 \mathrm{~g} / \mathrm{dL}(\mathrm{Hb}>11 \mathrm{~g}$; CTL $n=56$ and $\mathrm{RT}=57)$ as per the guidelines of the American Society of Nephrology (Kalantar-Zadeh and Aronoff, 2009). All exercise testing and prescription procedures were carried out following the guidelines of the American College of Sports Medicine (Pescatello et al., 2014). Moreover, the present study was conducted in accordance with the guidelines and regulations for exercise prescription for end-stage renal disease (Segura-Orti and Johansen, 2010).

\section{Resistance-Training Protocol}

The RT program was performed three times per week for 24 weeks and each training session took approximately $40 \mathrm{~min}$. Participants performed three sets of 8-12 repetitions with the training load monitored using the OMNI rating of perceived exertion (RPE) scale (Robertson et al., 2003). The rest interval between sets and exercises was $120 \mathrm{~s}$. Participants initially trained with a load corresponding to 8 repetitions at RPE of 5-6 for the first 12 weeks and 7-8 over the final 12 weeks. When the RPE indicated the load was too easy, we first increased the number of repetitions and, if the participant exceeded 12 repetitions, the load was increased.

The participants performed the pre-dialysis exercise approximately one hour before the start of their hemodialysis session. Each training session consisted of 12 exercises that included: chest press, squat, unilateral row, unilateral knee extension, unilateral knee flexion, unilateral shoulder press, hip thrust, unilateral biceps curl, unilateral hip adduction, unilateral elbow extension with dumbells, and seated calf raise. The e-Lastic (e-Sports Solutions, Brazil) cable was used to perform the chest press, unilateral row, and unilateral hip adduction exercises. For exercises performed with rubber bands, each repetition was counted by the application coupled to the e-Lastic dynamometer, allowing the measurement of the load used in each repetition.
Dumbbells were used for performance of the unilateral shoulder press, unilateral biceps curl, unilateral elbow extension. Weighted cuffs were used for the unilateral knee extension (wrapped at the ankle), unilateral knee flexion (wrapped at the ankle), hip thrust (positioned at the hips), and seated calf raise (wrapped across the quadriceps). In the squat exercise, due to the fragility of the lower limbs of hemodialysis patients, we chose to use only body weight to perform the squat exercise with just four repetitions at the beginning of the protocol. For upper limb exercises, we prioritized unilateral exercises as a conservative measure to preserve arteriovenous fistula. The RT routine consisted of three sets of 8-12 repetitions with two minutes of rest between sets at a cadence of two seconds for both concentric and eccentric phases. All RT sessions were individually supervised by a strength and conditioning professional.

\section{Body Composition}

All subjects were weighed on a mechanical scale (Filizola ${ }^{\circledR}$, São Paulo, Brazil), and height was measured with a stadiometer built into the scale (precision: $0.5 \mathrm{~cm}$ ). Waist circumference was assessed at the level of umbilicus using an anthropometric tape $\left(\right.$ Sanny ${ }^{\circledR}$, São Paulo, Brazil). Body fat and fat-free mass were measured using a Prodigy Advance Plus (LUNAR,Corp/General Electric; Madison, WI, United States) dual-energy X-ray absorptiometry (DXA) unit. Volunteers were asked to remove any metallic items they were wearing, such as rings, jewelry, belts, and watches (given that such objects affect the values of the estimated variables). Next, volunteers were placed in horizontal decubitus dorsal on the DXA table for full-body analysis. Before each use, the DXA equipment was calibrated according to the manufacturer's recommendations, and cut line adjustments were predefined. All analyses were performed by the same evaluator.

\section{Handgrip Strength}

Handgrip strength was measured with a hydraulic hand dynamometer (Jamar ${ }^{\circledR}$ - Sammons Preston, Bolingbrook, United States), according to the American Society of Hand Therapists' recommendations (Mathiowetz et al., 1984). Measurements were performed with participants in sitting position, elbow joint at $90^{\circ}$, forearm in a neutral position, and wrist between $0^{\circ}$ and $30^{\circ}$ of extension. The average of three attempts in the contralateral arm of the arteriovenous fistula, with 60 seconds rest between attempts, was registered in kgf. 


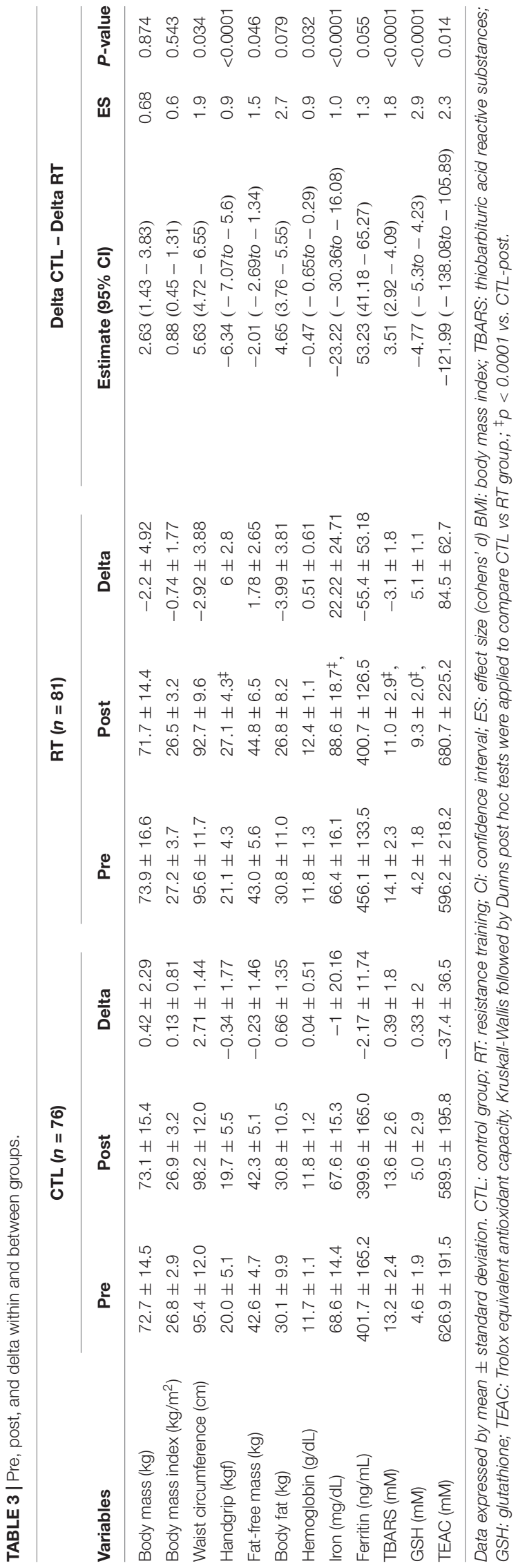

\section{Biochemical Analysis}

For lipid peroxidation, serum samples $(80 \mu \mathrm{L})$ were diluted in $320 \mu \mathrm{L}$ MiliQ $\mathrm{H} 2 \mathrm{O}$ (1:5), and then $1 \mathrm{~mL}$ of 5 trichloroacetic acid (TCA) $17.5 \%, \mathrm{pH} 2.0$, and $1 \mathrm{~mL}$ of thiobarbituric acid $0.6 \%, \mathrm{pH}$ 2.0 , were added, respectively. After homogenization, the samples were kept in a water bath for $30 \mathrm{~min}$ at $95^{\circ} \mathrm{C}$. The reaction was interrupted with the immersion of the microtubes in ice, the addition of $1 \mathrm{~mL}$ of TCA 70\%, $\mathrm{pH} 2.0$, and incubation for $20 \mathrm{~min}$ at room temperature. After centrifugation $(3,000 \mathrm{rpm}$ for $15 \mathrm{~min}$ ), the supernatant was removed and put in new microtubes and read by spectrophotometry at $540 \mathrm{~nm}$. The concentration of lipid peroxidation products was calculated using the molar extinction coefficient equivalent for malondialdehyde (MDA-equivalent $\left.=1.56 \cdot 105 \mathrm{M}^{-1} \mathrm{~cm}^{-1}\right)$. The coefficients of variability were $<6 \%$. Total antioxidant capacity was measured with a Trolox-equivalent assay kit (Quanti $\mathrm{Chrom}^{\circledR}$ Bio Assay Systems, CA, United States). Reduced form of glutathione (GSH) was measured from plasma using an assay kit (Sigma Aldrich ${ }^{\circledR}$, CA, United States) with a final spectrophotometric reading at $490 \mathrm{~nm}$. Briefly, in a 96-well microplate, $20 \mu \mathrm{L}$ of sample and $1,000 \mu \mathrm{L}$ of working reagent were added to each well. After homogenizing and incubating at $37^{\circ} \mathrm{C}$ for $5 \mathrm{~min}$, the absorbance was determined at $505 \mathrm{~nm}$ in a microplate reader (ELx800, Bio Tek, United States). For all antioxidant parameters, the intraassay coefficient of variation was $<4 \%$. Hematological profile was assessed by the automated ABX Micros 60 analyzer in a private and standard commercial laboratory.

\section{Subgroup Stratification}

Based on inferences from previous studies demonstrating anemia as a condition that influences peak oxygen uptake in CKD patients (Stray-Gundersen et al., 2016), we stratified the subgroups according to their serum hemoglobin levels $(\mathrm{Hb}<11$ or $\mathrm{Hb}>11)$. Therefore, control group (CTL) was stratified in CTL Hb $<11(n=20)$ and CTL Hb $>11(n=56)$; and RT group was stratified in $\mathrm{RT} \mathrm{Hb}<11(n=23)$ and RT Hb $>11(n=58)$.

\section{Statistical Analysis}

The primary endpoint of the present study was the effect of RT on strength and fat-free mass gain, and the secondary endpoint was the effect on redox balance. A third endpoint was to evaluate the effect of baseline hemoglobin levels on RT response. The normality and homogeneity of data were tested by the Kolmogorov-Smirnov and Levene tests, respectively. Data were expressed as medians (minimum - maximum) for baseline characteristics and means \pm standard deviations to pre- and post-exercise training. The effect size was determined using Cohens' $d$. Also, a 95\% confidence interval (CI) was presented for the mean differences ( $\Delta \mathrm{CTL}-\Delta \mathrm{RT})$ between groups. Given violations of normality, Chi-square and Mann-Whitney tests were used to analyze the baseline characteristics of individuals. Moreover, Mann-Whitney was also used to compare deltas (post - pre) of strength, fat-free mass gain and redox balance according to baseline $\mathrm{Hb}$. Anthropometry, strength, clinical patterns (hemoglobin, iron, and ferritin) and redox balance were compared at pre- and post-training by the Kruskal-Wallis test 


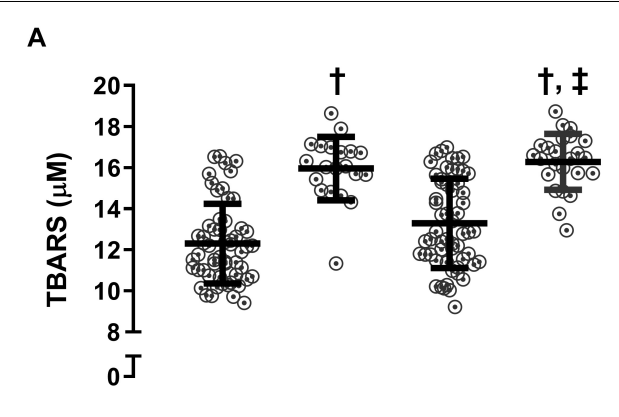

B
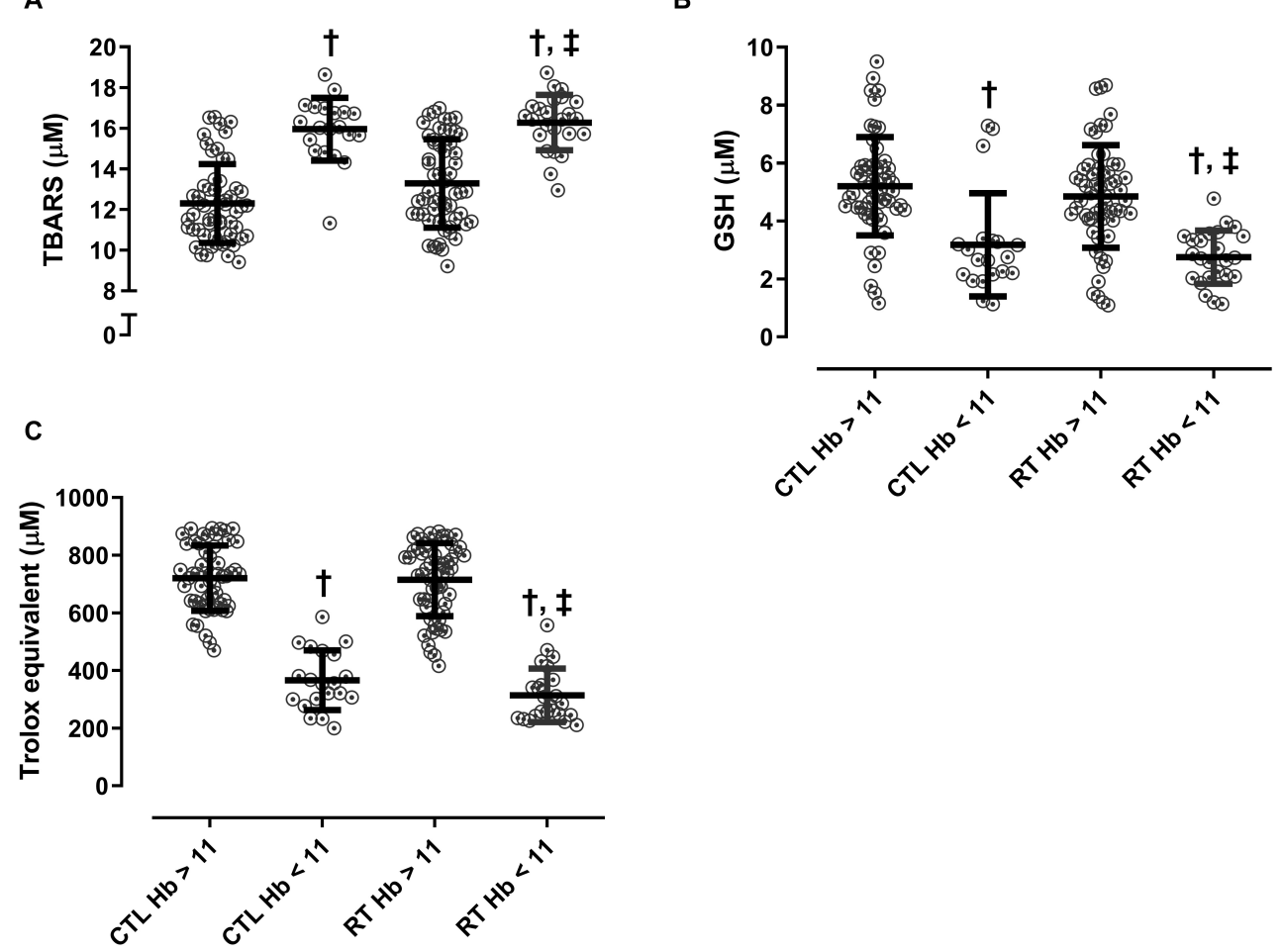

FIGURE 1 | Baseline redox balance according to hemoglobin stratification, $\mathrm{Hb}<11 \mathrm{~g} / \mathrm{dl}$ and $\mathrm{Hb}>11 \mathrm{~g} / \mathrm{dL}$. (A) TBARS, (B) GSH, and (C) trolox equivalent. TBARS: thiobarbituric acid reactive substances; GSH: glutathione; TEAC: Trolox equivalent antioxidant capacity; Hb: hemoglobin; CTL: control; RT: resistance training. Kruskal-Wallis test followed by Dunns' post hoc were applied to compare groups. ${ }^{\dagger} p<0.05$ vs. CTL Hb $>11,{ }^{\ddagger} p<0.05$ vs. RT Hb $>11$.

followed by Dunns' post hoc. Furthermore, Fishers exact test was performed to compare the proportions of the number of patients with $\mathrm{Hb}<11$ for both CTL and RT groups. Statistical significance was accepted at $p<0.05$. Statistical analyses were performed using the GraphPadPrism6.0 (San Diego, United States).

\section{RESULTS}

One hundred fifty-seven patients completed the intervention. As presented in Table 1, there were no differences between groups for baseline characteristics. the loads observed at the beginning and at the end of RT program for e-Lastic cable, dumbbells, and weighted cuffs are presented in Table 2.

The RT group displayed a greater improvement pre- to post $v s$ CTL handgrip strength and TBARS $(p<0.0001)$. RT also showed greater pre- to post changes $v s$ CTL in hemoglobin, iron and GSH $(p<0.0001)$. as displayed in Table 3.

Although RT promoted several improvements in patients with CKD, a considerable dispersion was noted in our data related to fat-free mass and redox balance. Considering that some patients started the protocol with a low concentration of hemoglobin $(<11 \mathrm{~g} / \mathrm{dL})$, which potentially could confound the data, we stratified the RT group according to baseline levels of hemoglobin to determine if lower levels of hemoglobin may have interfered with exercise-related benefits. Results showed that participants with lower hemoglobin levels at baseline presented lower markers of antioxidant defense and higher TBARS $(p<0.0001)$ as compared to participants with normal hemoglobin levels

TABLE 4 | Change percent for each group.

\begin{tabular}{lcccc}
\hline Variables & $\begin{array}{c}\mathbf{C T L} \\
\mathbf{H b}<\mathbf{1 1} \\
(\mathbf{\%})\end{array}$ & $\begin{array}{c}\mathbf{C T L} \\
\mathbf{H b}>\mathbf{1 1} \\
\mathbf{( \% )}\end{array}$ & $\begin{array}{c}\mathbf{R T} \\
\mathbf{H b}<\mathbf{1 1} \\
\mathbf{( \% )}\end{array}$ & $\begin{array}{c}\mathbf{R T} \\
\mathbf{H b}>\mathbf{1 1} \\
\mathbf{( \% )}\end{array}$ \\
\hline Body mass $(\mathrm{kg})$ & +0.7 & +0.5 & -4.7 & -2.3 \\
Body mass index $\left(\mathrm{kg} / \mathrm{m}^{2}\right)$ & +0.6 & +0.4 & -4.5 & -2.1 \\
Waist circumference $(\mathrm{cm})$ & +2.7 & +2.8 & -1 & -3.8 \\
Handgrip (kgf) & +1.5 & -2.8 & $\uparrow 23.9$ & $\uparrow 30.1$ \\
Fat-free mass $(\mathrm{kg})$ & +0.1 & -0.8 & +1.2 & +5.2 \\
Body fat $(\mathrm{kg})$ & +1.6 & +1.4 & $\downarrow 13.7$ & $\downarrow 12.6$ \\
Hemoglobin $(\mathrm{g} / \mathrm{dL})$ & -0.04 & -0.4 & $\uparrow 11.8$ & +1.9 \\
Iron $(\mu \mathrm{g} / \mathrm{dL})$ & +6.2 & -3.9 & $\uparrow 51.5$ & $\uparrow 26.6$ \\
Ferritin $(\mathrm{ng} / \mathrm{mL})$ & -1 & -0.3 & $\downarrow 18.3$ & $\downarrow 9.5$ \\
TBARS $(\mu \mathrm{M})$ & +0.9 & +4 & $\downarrow 21.2$ & $\downarrow 22.4$ \\
GSH $(\mu \mathrm{M})$ & +18.2 & +4.8 & $\uparrow 189.3$ & $\uparrow 104.5$ \\
TEAC $(\mu \mathrm{M})$ & -4.6 & -6 & $\uparrow 27.8$ & $\uparrow 11.7$ \\
\hline
\end{tabular}

$C T L$, control group; $R T$, resistance training; $H b$, hemoglobin; BMI, body mass index; TBARS, thiobarbituric acid reactive substances; GSH, glutathione; TEAC, Trolox equivalent antioxidant capacity.

+ non-significant increase. - non-significant decrease. $\uparrow$ significant increase $(P<0.05) . \downarrow$ significant decrease $(P<0.05)$. 

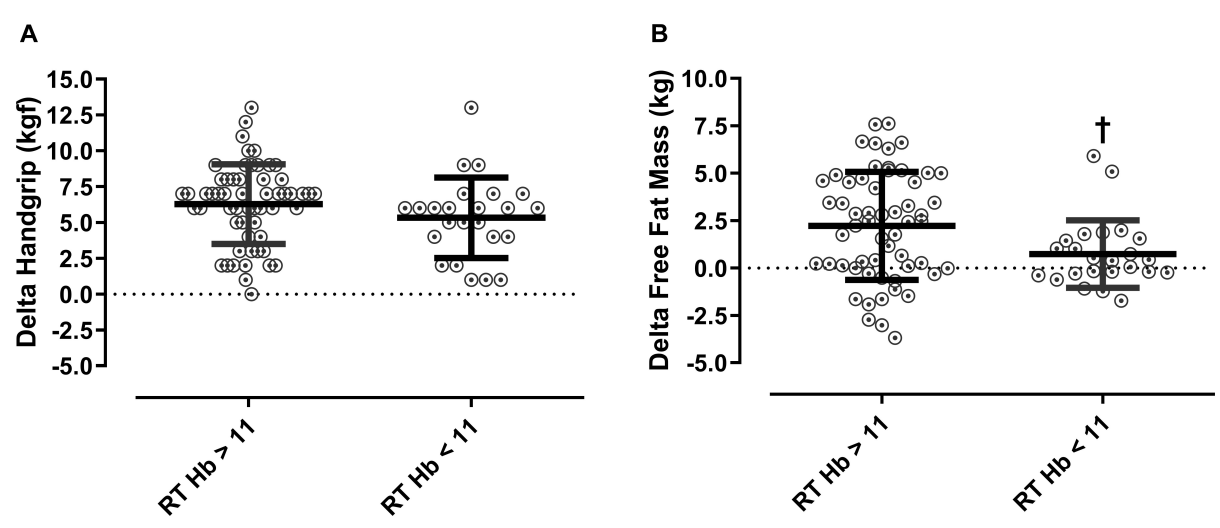

FIGURE 2 | Delta Handgrip strength, and fat-free mass according to baseline hemoglobin concentrations in the RT group. Mann-Whitney test was applied to compare deltas. (A) Delta of handgrip strength and (B) delta fat-free mass. Hb: hemoglobin; RT: resistance training ${ }^{\dagger} p<0.0001$ vs. $\mathrm{RT}$ Hb $>11$.

\section{A}

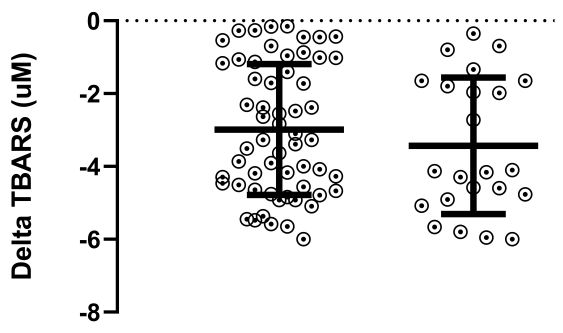

C

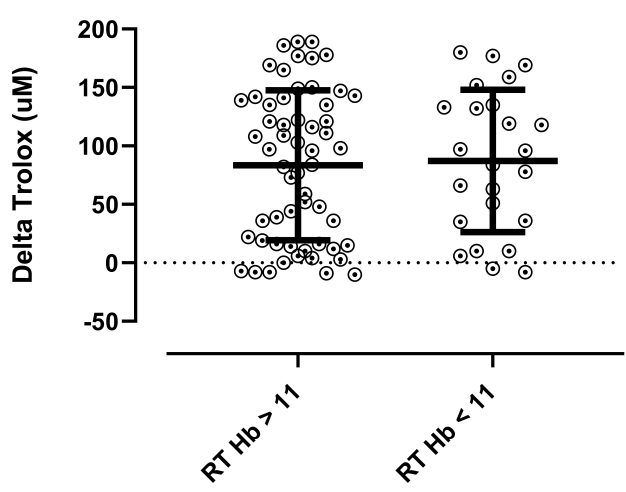

B

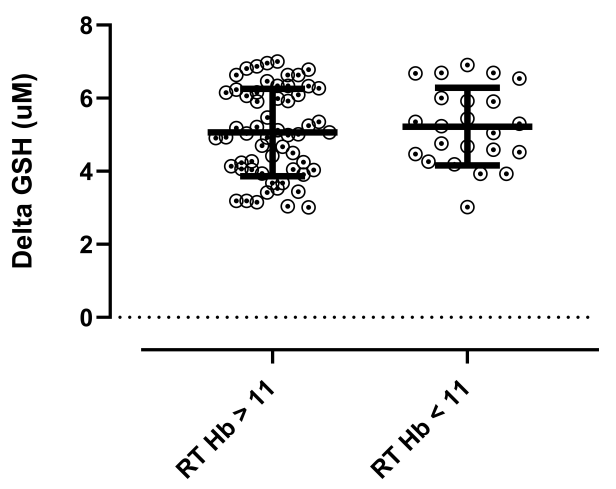

FIGURE 3 | Delta Redox balance according to baseline concentration of hemoglobin. (A) Delta TBARS, (B) delta GSH, and (C) delta trolox. TBARS: thiobarbituric acid reactive substances; GSH: glutathione; TEAC: Trolox equivalent antioxidant capacity; Hb: hemoglobin; RT: resistance training. Mann-Whitney test was applied to compare groups.

(Figure 1). It is worthy state that body composition and strength did not differ according to $\mathrm{Hb}$ levels at baseline. Moreover, the change percent for each group are presented in Table 4.

As observed in Figure 2, patients from the RT group with low baseline levels of hemoglobin levels had an attenuated effect of RT on fat-free mass as compared with patients with normal hemoglobin $(p<0.0001)$.

There was no difference in TBARS, GSH, and Trolox when comparing low versus normal hemoglobin levels $(p>0.05)$.
Described in Figure 3. The number of patients with $\mathrm{Hb}<11$ preand post- intervention are described on Table 5.

\section{DISCUSSION}

We sought to determine the effect of RT on body composition, redox balance, and handgrip strength in maintenance hemodialysis patients. Despite the significant improvement in fat-free mass, strength and redox balance, our data showed a 
huge variation in response to the training protocol. Therefore, based on inferences from previous studies demonstrating anemia as a condition that influences peak oxygen uptake in CKD patients (Stray-Gundersen et al., 2016), we stratified the subgroups according to their blood hemoglobin levels. The main findings of the present study demonstrated that RT improves body composition, strength, and redox balance in CKD patients. Moreover, when stratified by hemoglobin levels, we observed that the effect of RT on fat-free mass gain is dependent on hemoglobin concentration in maintenance hemodialysis patients.

Anemia is extremely common among patients on hemodialysis, and can accentuate symptoms associated with decreased kidney function, such as fatigue, oxidative stress dyspnea and reduced exercise tolerance (Ye et al., 2018), which could possibly affect the development of strength and muscle hypertrophy. Although the Kidney Disease Improving Global Outcomes (KDIGO) guidelines have been used for anemia control goals (Ye et al., 2018), little is known about the impact of the patient's initial hemoglobin level on the effects of physical training, especially for the objective of preventing sarcopenia and oxidative damage. Considering that sarcopenia and oxidative stress are constant issues in CKD, especially in maintenance hemodialysis patients (Johansen and Lee, 2015), the improvement of body composition and redox balance in the present study was an important finding for this population due to the strong risk for cachexia and frailty (Cheung et al., 2010; Von Haehling et al., 2017). As described by Johansen and Lee (2015), obesity is an increasing issue in end-stage renal patients; therefore, RT can be a potential interventional tool for the treatment/prevention of this condition, contributing to an enhanced body composition.

Hemodialysis patients have a strong susceptibility to acquire metabolic and nutrition complications which negatively affect handgrip strength (Garagarza et al., 2018). Garagarza et al. (2018) demonstrated that handgrip strength is highly correlated with lean tissue mass in hemodialysis patients. In this regard, a relevant finding of the present study is that RT promotes an increase in handgrip strength, which may prevent comorbidities related to muscle wasting (Von Haehling et al., 2017; Jhee et al., 2020). Nevertheless, when stratified by hemoglobin concentration, patients with anemia $(<11 \mathrm{~g} / \mathrm{dL}$ in hemoglobin) did not realize RT-induced improvements in fat-free mass. This finding may be explained by evidence that lower levels of hemoglobin negatively affect the cardio-renal axis by increasing inflammation and oxidative stress (Collister et al., 2017; Nuhu and Bhandari, 2018; Gafter-Gvili et al., 2019), thus leading to muscle wasting (Jo et al., 2012) and, consequently, an increase in overall risk of cardiovascular outcomes.

Historically, was observed that low hemoglobin decreased the exercise performance of health subjects (Kaepovich and Millman, 1942; Balke et al., 1954; Calbet et al., 2006). Also, blood hemoglobin seems to decline according to the progression of CKD (Clyne et al., 1994). Therefore, low hemoglobin in hemodialysis patients appears to be a relevant factor to decrease exercise capacity in this population. This condition might be related to increased oxidative stress and low renal function in patients with $\mathrm{Hb}<11$ which significantly impairs the muscle
TABLE 5 | Number of patients for each group pre- and post- intervention.

\begin{tabular}{lccc}
\hline Conditions & Pre & Post & Total \\
\hline $\mathrm{CTL} \mathrm{Hb}<10$ & 20 & 19 & 39 \\
$\mathrm{CTL} \mathrm{Hb}>10$ & 56 & 57 & 113 \\
$\mathrm{RT} \mathrm{Hb}<10$ & 23 & 4 & 27 \\
$\mathrm{RT} \mathrm{Hb}>10$ & 58 & 77 & 135
\end{tabular}

CTL, control group; RT, resistance training group; Hb, hemoglobin.

mass gain (Moylan and Reid, 2007). However, further studies should evaluate the possible mechanisms related to the low hemoglobin impairing fat-free mass after RT. Another key finding of the present study is that just 4 patients continued with $\mathrm{Hb}<11$ after the intervention. Demonstrating that RT should be part of the treatment of anemia in this population. To the best of our knowledge, this is the first study that verified the influence of low hemoglobin on a long-term RT protocol, being clinically relevant in the prevention and treatment of CKDrelated comorbidities.

A notable limitation of the present study is that we did not perform tissue biopsy analysis to investigate the iron balance before and after RT, while this data brings mechanistic insights of RT generating improvements in muscle mass, strength, body composition and redox balance in maintenance hemodialysis patients. However, our results demonstrated that muscle mass may depend on normal levels of hemoglobin (>11ng/L). In this regard, it seems that not only is aerobic training impaired by baseline hemoglobin levels (Stray-Gundersen et al., 2016), but resistance training as well, perhaps effectuated by different mechanisms. Furthermore, food intake, were not controlled in the present analysis. We recognize it as a potential limitation of the present study and encourage further studies to control this variable. Finally, we also encourage further studies to investigate the influence of the level of physical activity as a possible factor the influences RT response in this population.

The present study expands our knowledge of how RT affects body composition, handgrip strength and redox balance in maintenance hemodialysis patients. Given our findings, practitioners should seek to treat those with anemia or lower concentrations of hemoglobin before beginning a RT intervention in this population, as these conditions impair fatfree mass gain. Implementation of these findings can contribute to a better clinical application of RT in patients with CKD.

In conclusion, six months of RT improved clinical patterns, such as hemoglobin, iron, body composition and redox balance in $\mathrm{HD}$ patients. In addition, a low hemoglobin concentration impairs the exercise-benefits on fat-free mass. Nevertheless, RT should be recommended as a nonpharmacological interventional tool to improve the clinical status of this population.

\section{DATA AVAILABILITY STATEMENT}

The raw data supporting the conclusions of this article will be made available by the authors, without undue reservation. 


\section{ETHICS STATEMENT}

The studies involving human participants were reviewed and approved by Catholic University of Brasilia Ethics Committee: 23007319.0.0000.0029. The patients/participants provided their written informed consent to participate in this study.

\section{AUTHOR CONTRIBUTIONS}

VS, HC, and TR: conceptualization and project administration. VS, RN, LD, AR, CS, and TR: data curation. VS, HC, MS, LD, $\mathrm{AR}$, and TR: formal analysis. VS, FH, HS, and MM: funding

\section{REFERENCES}

Astor, B. C., Muntner, P., Levin, A., Eustace, J. A., and Coresh, J. (2002). Association of kidney function with anemia: the third national health and nutrition examination survey (1988-1994). Arch. Intern. Med. 162, 1401-1408. doi: 10.1001/archinte.162.12.1401

Balke, B., Grillo, G. P., Konecci, E. B., and Luft, U. C. (1954). Work capacity after blood donation. J. Appl. Physiol. 7, 231-238. doi: 10.1152/jappl.1954.7. 3.231

Bissinger, R., Bhuyan, A. A. M., Qadri, S. M., and Lang, F. (2019). Oxidative stress, eryptosis and anemia: a pivotal mechanistic nexus in systemic diseases. FEBS J. 286, 826-854. doi: 10.1111/febs.14606

Calbet, J. A., Lundby, C., Koskolou, M., and Boushel, R. (2006). Importance of hemoglobin concentration to exercise: acute manipulations. Respir. Physiol. Neurobiol. 151, 132-140. doi: 10.1016/j.resp.2006. 01.014

Cheema, B., Abas, H., Smith, B., O'sullivan, A., Chan, M., Patwardhan, A., et al. (2007). Progressive exercise for anabolism in kidney disease (PEAK): a randomized, controlled trial of resistance training during hemodialysis. J. Am. Soc. Nephrol. 18, 1594-1601. doi: 10.1681/asn.2006121329

Chen, J. L., Godfrey, S., Ng, T. T., Moorthi, R., Liangos, O., Ruthazer, R., et al. (2010). Effect of intra-dialytic, low-intensity strength training on functional capacity in adult haemodialysis patients: a randomized pilot trial. Nephrol. Dial. Transplant. 25, 1936-1943. doi: 10.1093/ndt/gfp739

Cheung, W. W., Paik, K. H., and Mak, R. H. (2010). Inflammation and cachexia in chronic kidney disease. Pediatr. Nephrol. 25, 711-724. doi: 10.1007/s00467009-1427-z

Clyne, N., Jogestrand, T., Lins, L. E., and Pehrsson, S. K. (1994). Progressive decline in renal function induces a gradual decrease in total hemoglobin and exercise capacity. Nephron 67, 322-326. doi: 10.1159/000187987

Collister, D., Rigatto, C., and Tangri, N. (2017). Anemia management in chronic kidney disease and dialysis: a narrative review. Curr. Opin. Nephrol. Hypertens. 26, 214-218. doi: 10.1097/mnh.0000000000000317

Corrêa, H. L., Moura, S. R. G., Neves, R. V. P., Tzanno-Martins, C., Souza, M. K., Haro, A. S., et al. (2020). Resistance training improves sleep quality, redox balance and inflammatory profile in maintenance hemodialysis patients: a randomized controlled trial. Sci. Rep. 10:11708.

Gafter-Gvili, A., Schechter, A., and Rozen-Zvi, B. (2019). Iron Deficiency Anemia in Chronic Kidney Disease. Acta Haematol. 142, 44-50.

Garagarza, C., Flores, A. L., and Valente, A. (2018). Influence of Body Composition and Nutrition Parameters in Handgrip Strength: Are There Differences by Sex in Hemodialysis Patients? Nutr. Clin. Pract. 33, 247-254. doi: 10.1177/ 0884533617725512

Jhee, J. H., Joo, Y. S., Han, S. H., Yoo, T. H., Kang, S. W., and Park, J. T. (2020). High muscle-to-fat ratio is associated with lower risk of chronic kidney disease development. J. Cachexia Sarcopenia Muscle 11, 726-734. doi: 10.1002/jcsm. 12549

Jo, E., Lee, S.-R., Park, B.-S., and Kim, J.-S. (2012). Potential mechanisms underlying the role of chronic inflammation in age-related muscle wasting. Aging Clin. Exp. Res. 24, 412-422. acquisition. VS, HC, DC, and TR: investigation. VS, DC, JP, and TR: methodology. VS, HC, BS, and TR: roles/writing original draft. VS, HC, JP, BS, and TR: writing - review and editing. All authors contributed to the article and approved the submitted version.

\section{FUNDING}

This study was financed in part by the Coordenação de Aperfeiçoamento de Pessoal de Nível Superior-Brazil (CAPES)-Finance Code 001.

Johansen, K. L., and Lee, C. (2015). Body composition in chronic kidney disease. Curr. Opin. Nephrol. Hypertens. 24:268.

Kaepovich, P. V., and Millman, N. (1942). Athletes as blood donors. Res. Quart. Am. Associat. Health 13, 166-168. doi: 10.1080/10671188.1942. 10624742

Kalantar-Zadeh, K., and Aronoff, G. R. (2009). Hemoglobin variability in anemia of chronic kidney disease. J. Am. Soc. Nephrol. 20, 479-487. doi: 10.1681/asn. 2007070728

Kopple, J. D. (2001). National kidney foundation K/DOQI clinical practice guidelines for nutrition in chronic renal failure. Am. J. Kidney Dis. 37, S66-S70. doi: 10.1053/ajkd.2001.20748

Kopple, J. D., Wang, H., Casaburi, R., Fournier, M., Lewis, M. I., Taylor, W., et al. (2007). Exercise in maintenance hemodialysis patients induces transcriptional changes in genes favoring anabolic muscle. J. Am. Soc. Nephrol. 18, 2975-2986. doi: 10.1681 /asn.2006070794

Marinho, S. M., Mafra, D., Pelletier, S., Hage, V., Teuma, C., Laville, M., et al. (2016). In Hemodialysis Patients, Intradialytic Resistance Exercise Improves Osteoblast Function: A Pilot Study. J. Ren. Nutr. 26, 341-345. doi: 10.1053/j. jrn.2016.03.002

Mathiowetz, V., Weber, K., Volland, G., and Kashman, N. (1984). Reliability and validity of grip and pinch strength evaluations. J. Hand Surg. Am. 9, 222-226. doi: 10.1016/s0363-5023(84)80146-x

Mcclellan, W., Aronoff, S. L., Bolton, W. K., Hood, S., Lorber, D. L., Tang, K. L., et al. (2004). The prevalence of anemia in patients with chronic kidney disease. Curr. Med. Res. Opin. 20, 1501-1510. doi: 10.1185/030079904X2763

Moura, S. R. G., Corrêa, H. L., Neves, R. V. P., Santos, C. A. R., Neto, L. S. S., Silva, V. L., et al. (2020). Effects of resistance training on hepcidin levels and iron bioavailability in older individuals with end-stage renal disease: A randomized controlled trial. Exp. Gerontol. 139:111017. doi: 10.1016/j.exger.2020.11 1017

Moylan, J. S., and Reid, M. B. (2007). Oxidative stress, chronic disease, and muscle wasting. Muscle Nerve 35, 411-429. doi: 10.1002/mus.20743

Neves, R. V. P., Corrêa, H. L., De Deus, L. A., Reis, A. L., Souza, M. K., Simoes, H. G., et al. (2020). Dynamic not isometric training blunts osteo-renal disease by the sclerostin/FGF23/Klotho axis in maintenance hemodialysis patients: a randomized clinical trial. J. Appl. Physiol. Preprint.

Nuhu, F., and Bhandari, S. (2018). Oxidative stress and cardiovascular complications in chronic kidney disease, the impact of anaemia. Pharmaceuticals 11:103. doi: 10.3390/ph11040103

Pescatello, L. S., Riebe, D., and Thompson, P. D. (2014). ACSM's guidelines for exercise testing and prescription. Philadelphia: Lippincott Williams \& Wilkins.

Robertson, R. J., Goss, F. L., Rutkowski, J., Lenz, B., Dixon, C., Timmer, J., et al. (2003). Concurrent validation of the OMNI perceived exertion scale for resistance exercise. Med. Sci. Sports Exerc. 35, 333-341. doi: 10.1249/01.mss. $0000048831.15016 .2 \mathrm{a}$

Rosa, C., Nishimoto, D. Y., Souza, G. D. E., Ramirez, A. P., Carletti, C. O., Daibem, C. G. L., et al. (2018). Effect of continuous progressive resistance training during hemodialysis on body composition, physical function and quality of life in endstage renal disease patients: a randomized controlled trial. Clin. Rehabil. 32, 899-908. doi: 10.1177/0269215518760696 
Segura-Orti, E., and Johansen, K. L. (2010). Exercise in end-stage renal disease. Semin. Dialys. 23, 422-430. doi: 10.1111/j.1525-139x.2010.00 766.x

Sietsema, K. E., Amato, A., Adler, S. G., and Brass, E. P. (2004). Exercise capacity as a predictor of survival among ambulatory patients with end-stage renal disease. Kidney Int. 65, 719-724. doi: 10.1111/j.1523-1755.2004.00411.x

Song, W. J., and Sohng, K. Y. (2012). Effects of progressive resistance training on body composition, physical fitness and quality of life of patients on hemodialysis. J. Korean Acad. Nurs. 42, 947-956. doi: 10.4040/jkan.2012.42.7. 947

Stray-Gundersen, J., Howden, E. J., Parsons, D. B., and Thompson, J. R. (2016). Neither hematocrit normalization nor exercise training restores oxygen consumption to normal levels in hemodialysis patients. J. Am. Soc. Nephrol. 27, 3769-3779. doi: 10.1681/asn.2015091034

Suresh, K. (2011). An overview of randomization techniques: an unbiased assessment of outcome in clinical research. J. Hum. Reproduct. Sci. 4:8. doi: 10.4103/0974-1208.82352

Von Haehling, S., Ebner, N., Dos Santos, M. R., Springer, J., and Anker, S. D. (2017). Muscle wasting and cachexia in heart failure: mechanisms and therapies. Nat. Rev. Cardiol. 14:323. doi: 10.1038/nrcardio.20 17.51
Wong, M. M., Tu, C., Li, Y., Perlman, R. L., Pecoits-Filho, R., Lopes, A. A., et al. (2019). Anemia and iron deficiency among chronic kidney disease Stages 3$5 \mathrm{ND}$ patients in the chronic kidney disease outcomes and practice patterns study: often unmeasured, variably treated. Clin. Kid. J. 13, 613-624. doi: 10. 1093/ckj/sfz091

Ye, Y., Liu, H., Chen, Y., Zhang, Y., Li, S., Hu, W., et al. (2018). Hemoglobin targets for the anemia in patients with dialysis-dependent chronic kidney disease: a meta-analysis of randomized, controlled trials. Renal Failure 40, 671-679. doi: 10.1080/0886022x.2018.1532909

Conflict of Interest: The authors declare that the research was conducted in the absence of any commercial or financial relationships that could be construed as a potential conflict of interest.

Copyright (C) 2021 da Silva, Corrêa, Neves, Deus, Reis, Souza, dos Santos, de Castro, Honorato, Simões, Moraes, Schoenfeld, Prestes and Rosa. This is an open-access article distributed under the terms of the Creative Commons Attribution License (CC BY). The use, distribution or reproduction in other forums is permitted, provided the original author(s) and the copyright owner(s) are credited and that the original publication in this journal is cited, in accordance with accepted academic practice. No use, distribution or reproduction is permitted which does not comply with these terms. 